\title{
Actividades para fomentar la fluidez en la expresión escrita
}

JUAN RUIZ HERRERO

Accem-Valencia

jruizh@accem.es

Resumen: La fluidez se concibe y se practica en el aula fundamentalmente como un atributo de las destrezas productivas orales, mientras la instrucción de la expresión escrita tiende a quedar dominada por preocupaciones de carácter formal. Se presentan, pues, consideraciones para fundamentar actividades que faciliten el desarrollo de la fluidez a la hora de escribir y se describen dos secuencias para ejemplificarlo.

Palabras clave: fluidez, expresión, escrita, interacción.

\section{Fostering fluency development though written activities in the language classroom}

Abstract: Fluency is often conceptualized and practiced as an exclusive feature of oral abilities in the language classroom, while written instruction is often exclusively focalized in formal considerations. The article presents a set of principles which might foster fluency development in written tasks and provides two whole activity sequences illustrating said aims.

Key words: fluency, expression, written, interaction

\section{Introducción}

En el ámbito de la didáctica de lenguas la fluidez se presenta como un parámetro que se reconoce, valora y computa con cierta familiaridad desde los primeros pasos prácticos del docente. En tanto que manifestación directa de la facilidad del aprendiente a la hora de expresarse, existe una tradición muy cimentada desde la generalización de los enfoques comunicativos de incentivar la producción de un caudal lingüístico de un cierto volumen que mantenga un determinado ritmo, a expensas de la corrección del mensaje, como reflejo del desplazamiento generalizado del foco de enseñanza desde la forma al significado. Es cierto, no obstante, que la fluidez se tiende a percibir y predicar como atributo exclusivo de las destrezas productivas orales. En las siguientes páginas, así, se cuestionará la pertinencia de esta perspectiva restrictiva y se recopilarán elementos que puedan servir para potenciar la fluidez en actividades de expresión escrita. A partir de esta caracterización, se propondrán dos secuencias, entendidas como ilustraciones de una técnica susceptible de ser extrapolada a diferentes realidades docentes y niveles de aprendizaje.

\section{Hablemos de fluidez}

No resulta tarea fácil comenzar la propuesta echando mano de una definición canónica de fluidez, aunque no precisamente a causa de carencias al respecto. Definiciones de fluidez existen por decenas, pero la mayor parte de contribuciones que abordan la 
temática señalan ante todo precisamente la dificultad de atenerse a una formulación canónica que sirva de planteamiento consensuado. A medida que nos adentramos en la bibliografía relacionada, emergen algunos puntos recurrentes. En primer lugar, nos encontramos ante un término ubicuo, al que se recurre de forma permanente desde disciplinas muy diferentes, dentro y fuera de la Didáctica de lenguas, dentro y fuera de la Lingüística aplicada. Segundo, que se trata de un constructo multidimensional, que si bien se relaciona fundamentalmente con toda una serie de aspectos cognitivos que facilitan la producción del discurso, está estrechamente ligado con el efecto que se produce en el receptor y la facilidad y el esfuerzo que se requieren para descodificar el mensaje (Fraser 2014). Por último, su uso, si bien está generalizado, lo preside fundamentalmente la intuición, es decir, nos referimos a la fluidez y creemos reconocerla, basándonos más bien en aquello que pensamos entender como tal, más que en la ilustración de una realidad que seamos capaces de enunciar y describir (Dormer 2016). El resultado de una exploración bibliográfica de este tipo resulta, así, particularmente frustrante: no sólo no se consigue obtener una idea más ajustada de qué es la fluidez, sino que la sensación resultante es que nadie sabe con certeza qué es.

Y sin embargo, tal como señalábamos, las definiciones de fluidez abundan. Citemos algunas de las más manejables: la capacidad para producir discurso a un ritmo normal sin interrupción (Skehan 2009); la producción de lengua en tiempo real sin pausas ni dudas (Ellis \& Barkhuizen 2005) o la velocidad y la facilidad en la enunciación oral (Lennon 2000). Como se puede comprobar, todas estas definiciones, parten de un mismo punto, a saber, la conceptualización de la fluidez como un atributo exclusivo de la expresión oral. No en vano, se coincide con la percepción que tanto desde docentes como estudiantes de lenguas se posee del término. Así, por ejemplo, la mayoría ha estado de un lado o de otro de un examen certificativo de lenguas- esto es, presentándose al mismo o administrándolo- y reconoce "fluidez" como uno de los tres o cuatro criterios de evaluación que habitualmente se contemplan en la prueba oral. ¿Está en todo caso fundamentada una creencia así?

Recurramos en este punto a la definición que proporciona el profesor neozelandés Paul Nation, que contempla la fluidez como la facilidad para acceder al conocimiento lingüístico existente, así como para producir a partir del mismo (Nation 2014). Se percibe aquí un salto cualitativo importante, puesto que la fluidez se relaciona fundamentalmente con la capacidad de movilizar un conocimiento determinado para resolver una necesidad determinada, normalmente en relación con algún tipo de requerimiento temporal. Esto es, si bien está más que justificada la utilización del término para referirse a la expresión oral, no hay ningún motivo para no hacer lo propio con el resto de destrezas. Resulta, así, legítimo y apropiado reflexionar acerca de la fluidez en relación con las destrezas receptivas, puesto que haríamos alusión a la facilidad con la que se recurre al conocimiento lingüístico existente para procesar satisfactoriamente un mensaje determinado. El propio Paul Nation, de hecho, ha firmado numerosas contribuciones centradas en actividades que sirven para fomentar la fluidez en la comprensión lectora, esto es, para leer de forma más eficaz, aumentando las líneas, párrafos o páginas que se pueden entender en un tiempo determinado. Asimismo, desde esta perspectiva, tiene sentido plantear la fluidez en la expresión escrita para interesarnos por la facilidad con la que se produce discurso a través de un canal no oral. 
Se trata, de hecho, de un ámbito de investigación que posee ya una cierta trayectoria y que ha acumulado a lo largo de las últimas décadas un número razonable de contribuciones experimentales. La mayor parte de ellas adoptan perspectivas basadas en el producto. Son por lo general diseños que contabilizan la extensión de la producción normalmente antes y después de una intervención determinada y para los cuales se establecen toda una serie de indicadores que reinterpretan los atributos esenciales de la fluidez expresiva oral para analizar una producción escrita determinada (Fraser 2014). Se trata, en todo caso, de una perspectiva eminentemente cuantitativa que obvia tanto el contenido del escrito como el procedimiento de escritura. Por su parte, los estudios centrados en el proceso estipulan toda una serie de indicadores para analizar precisamente cómo se escriben los textos, para lo cual se graba a las personas a medida que van componiendo sus escritos (Abdel Latif 2014).

No es en cualquier caso el cometido de estas páginas proporcionar un estado de la cuestión detallado. Nos limitaremos en todo caso a referirnos a una investigación concreta, la publicada en 2014 por Theron Muller. No se trata en absoluto de una contribución puntera o de particular significancia en el ámbito, antes al contrario, hablamos de una propuesta que se realizó a una escala reducida, con unas intenciones humildes y que en la conclusión el propio autor reconoce como eminentemente fallida. No obstante, parte de unas reflexiones análogas a las que impulsaron la creación de las secuencias que aquí se presentan y con las que buena parte de docentes con un interés particular en la cuestión pueden identificarse. El experimento en cuestión se realizó en escuelas secundarias de Japón. Muller describe una realidad de aprendizaje presidida por la preparación de exámenes certificativos en la que la expresión escrita se entiende y se practica fundamentalmente como un ejercicio para demostrar la correcta utilización de toda una serie de estructuras y léxico. El autor se pregunta, así, acerca de la relación competitiva entre corrección y fluidez frecuentemente abordada desde la perspectiva de la expresión oral. Así, si se transmite al aprendiente que lo esencial a la hora de escribir es mantener un elevado nivel de corrección, se da una idea de lo irrelevante que resulta la fluidez al respecto y no se dedica esfuerzo alguno para desarrollarla en el aula. Del mismo modo, entiende, si se relativiza la importancia que lo correcto posee a la hora de enseñar a escribir y computar el resultado, se abrirán espacios y perspectivas para incidir en la trasmisión del mensaje y la facilidad de expresión.

Es ahora cuando resulta oportuno enlazar con otra contribución de cierto renombre, la de John Truscott, que en 1996 causó una considerable controversia en el ámbito de la Didáctica de la expresión escrita cuando afirmó de forma nada ambigua que "la corrección gramatical no tiene lugar alguno en los cursos de expresión escrita en L2 y debería abandonarse" (p.328). Argumentaba que en ningún momento se había podido establecer que las intervenciones centradas en la forma condujeran a ningún tipo de mejora en la capacidad de escribir de quienes la recibían. Entendía, por el contrario, que la corrección se desarrollaba de forma probable a través de una experiencia extensiva con la lengua meta y que, por lo tanto, lo pertinente desde un punto de vista didáctico era maximizar esa experiencia proporcionando el mayor número de oportunidades posibles para escribir. No daba a entender de esta forma que no hubiera justificación para una respuesta docente a los escritos producidos por los estudiantes, pero indicaba que ésta debía centrarse en cuestiones como el contenido, la organización o la claridad de la exposición, de tal forma que se transmitieran indicaciones rentables que facilitaran la producción de significado. La controversia suscitada queda convenientemente 
ilustrada con la serie de respuestas cruzadas que Truscott mantuvo con una de las principales autoras de estudios centrados en retroalimentación, Dana Ferris (1999). En cualquier caso, aquí nos limitaremos a señalar que sin compartir necesariamente la tajante exclusión de cualquier retroalimentación centrada en la forma propuesta por Truscott, fue en gran parte la lectura de sus propuestas la que guió la experimentación docente con este tipo de actividades.

\section{Actividades para desarrollar la fluidez de la expresión escrita}

Nation (2014) propone una serie de condiciones que deben reunir- si no todas, si el mayor número posible- aquellas actividades que tengan como objetivo desarrollar la fluidez, independientemente de la destreza comunicativa en la que se centren:

1. Deben estar centradas en el significado.

2. Tienen que versar sobre un contenido que resulte familiar para el aprendiente, de tal forma que se pueda dirigir lo esencial del esfuerzo cognitivo a la descodificación o codificiación eficaz de mensajes.

3. Ha de existir algún tipo de presión o incentivo para acelerar la cantidad de texto procesado o producido.

4. Deben procurar una abundancia de input, en el caso de las actividades centradas en promover la fluidez de las destrezas receptivas, o de output, en las que se centran en las destrezas productivas.

A partir de lo anterior, planteamos cinco condiciones que servirán para fundamentar la técnica aquí propuesta. Incidimos en que se trata de definir una técnica apoyada en una serie de elementos fijos susceptibles de ser manipulados para proponer una cantidad indefinida de secuencias, ajustadas en cada caso a unos objetivos comunicativos determinados y al público meta correspondiente.

a. Interacción: las actividades escritas tendrán un carácter fundamentalmente interactivo, de tal modo que el discurso producido se entienda dentro de una comunicación destinada a ser leída por otras personas, así como una respuesta a aquellas palabras que se han dirigido al aprendiente. Las secuencias requerirán, pues, escribir, pero también leer y a partir de ahí, contestar.

b. Géneros textuales electrónicos: redundando en la necesidad de cercanía apuntada más arriba, las secuencias aquí propuestas adoptarán formatos textuales extraídos de diferentes tipos de comunicación telemática. En la actualidad un gran número de hablantes recurre al canal escrito fundamentalmente para componer y enviar textos a través de servicios de mensajería instantánea, correo electrónico y diferentes redes sociales. Resulta, pues, conveniente capitalizar todo el conocimiento que un porcentaje considerable de los aprendientes posee acerca del formato y los parámetros comunicativos de determinados géneros textuales para propiciar una mayor producción de discurso.

c. Cercanía temática: a partir del mismo planteamiento, se tratará de explotar no sólo la familiaridad con el formato sino también con el contenido. Así, las actividades plantearán temáticas que resulten conocidas y de interés, asuntos 
acerca de los cuales haya experiencias que compartir u opiniones que defender. La elección temática resultará facilitada por el mayor conocimiento que se posea acerca del grupo o de la sociedad meta, puesto que se contará de este modo con un criterio más informado para identificar y descartar opciones. Así, cuanto mayor resulte su heterogeneidad- a nivel de nacionalidad, edad, perfiles profesionales y objetivos lingüísticos- más ardua será la tarea selectiva.

d. Retroalimentación de contenido: a partir de la posición defendida por John Truscott, las secuencias propuestas recurrirán a la retroalimentación de contenido como modo principal de respuesta a los textos producidos, en la medida que posibilitarán la continuación de un intercambio comunicativo más allá de la primera producción escrita. No significa en este caso que se renunciará a cualquier tipo de respuesta centrada en la forma, pero sí que este nivel de preocupación se relegará a un plano secundario o se postergará a un momento posterior.

e. Uso dinámico del espacio: se plantearán dinámicas que traduzcan la interacción en desplazamientos a través del aula y diferentes ocupaciones del espacio a medida que progresa la secuencia. Se atiende así a diversas consideraciones. En primer lugar, desde un punto de vista más general, para recoger las afirmaciones de diferentes autores que relacionan de forma positiva el recurso al movimiento en planteamientos didácticos con la generación de condiciones cognitivas que favorecen la atención y el aprendizaje (Ratey 2001; Jensen 1998). Pero al mismo tiempo se trata de subvertir la imagen generalizada de la actividad escrita en el aula de lengua como algo estático e individual, que transcurre plúmbeamente en la soledad del pupitre.

\section{Una propuesta}

A partir de lo anterior, pasamos a detallar el procedimiento de dos secuencias destinadas a dos niveles distintos. Ambas se concibieron y se llevaron al aula durante el año académico 2015/2016 en el Instituto Cervantes de Beirut, elemento en el que conviene incidir puesto que la aproximación temática a diferentes realidades culturales libanesas permea ambas.

\subsection{Secuencia 1: el Líbano de antes de la guerra}

La primera secuencia se planteó para un nivel A2, dentro de una unidad didáctica centrada en la descripción de una época anterior, que presentaba como exponente formal principal la introducción del uso y forma del Pretérito imperfecto de indicativo. Se planteó como tarea final en sustitución de la propuesta por el manual y su tiempo de realización se dividió en dos sesiones: 45 minutos para la primera y unos 20 para la siguiente.

La primera sesión arrancó con un estímulo meramente visual, en concreto un vídeo con información turística sobre Líbano de finales de los años sesenta que mostraba diferentes instalaciones hoteleras, puntos de interés y actividades de ocio presentadas para los visitantes potenciales. No poseía ningún tipo de contenido lingüístico en lengua alguna y su interés residía exclusivamente en mostrar toda una serie de imágenes que activaban conocimientos y percepciones previas sobre el tema en cuestión. Procede aquí 
introducir un breve paréntesis: Líbano conoció entre 1975 y 1990 una cruenta guerra civil que desgarró la pequeña república mediterránea en sucesivas olas de combates donde a las líneas de conflicto locales ideológicas y confesionales se sumaban sucesivas intervenciones exteriores y las correspondientes fracturas e intereses estratégicos regionales. El Líbano de principios de los años setenta, la antesala del destructivo conflicto nacional, se presenta así en el discurso común como la época dorada del país, un momento mitificado en el que habría reinado la prosperidad y en el que se generalizó el afectado título de "Suiza del Medio Oriente". Así, si bien es cierto que la mayor parte de estudiantes del grupo no poseía recuerdo alguno directo de una época muy anterior a su nacimiento, su evocación melancólica posee un carácter tan ritual en el discurso común que cualquier persona posee un considerable bagaje de nociones al respecto.

Acto seguido se presentaba la tarea (Anexo 1). Se indicaba a los estudiantes que habían encontrado en un grupo de Facebook de hispanohablantes en Líbano un mensaje de una joven que llevaba unos meses instalada en el país y a la que le habían llamado la atención las numerosas alusiones acerca de la época de antes de la guerra como referente positivo por defecto en comparación con la actualidad. Inquiría, así, acerca de lo apropiado de tal afirmación y se invitaba a los aprendientes a que proporcionaran una respuesta. Al apoyarse en un formato conocido- el mensaje de Facebook- no sólo se transmitían de forma implícita determinadas instrucciones sobre el género y la naturaleza interactiva del mismo, sino que también se favorecía la determinación de límites de espacio y de tiempo, puesto que los usuarios de la red social saben que un mensaje de esa naturaleza, por elaborado que pretenda ser, no puede exceder un número de líneas, más allá del cual nadie se molestará en leerlo. Se indicó, de este modo, un tiempo aproximado de veinte minutos para componer el texto.

A medida que cada una de las doce personas que componía el grupo iba terminando, su texto se pegaba con celo en la pared, que así pasaba a convertirse en un muro de Facebook, retomando su terminología propia. Acto seguido se facilitaba a cada una pequeña reproducción en papel del icono con la mano que en el código visual de la red social simboliza la aprobación, el mensaje de "me gusta". Se indicaba que se contaba ahora con diez minutos para pasear por la clase, leer las diferentes respuestas proporcionadas por los compañeros y asignar el signo de "me gusta" a aquella que se considerara más acertada. Esta dinámica conducía a un resultado en la forma de una propuesta ganadora, lo cual permitía cerrar la sesión con diferentes comentarios acerca de lo que había motivado la elección de cada quien.

Para la siguiente sesión se recogieron los textos compuestos y se procedió a responder. Se incluyeron comentarios relativos a la forma, pero atendiendo a dos consideraciones. En primer lugar, se trató de una respuesta selectiva, es decir, se intervino sólo a propósito de aquellas cuestiones que se consideraban más pertinentes por su incidencia comunicativa o por relacionarse con cuestiones abordadas a lo largo del curso en cuestión. Y en segundo lugar, la respuesta no fue explícita, es decir, no se proporcionó la alternativa correcta relacionada con cada error, sino que simplemente se señaló utilizando el código de marcas que se había aplicado a lo largo de todo el curso y con el cual los aprendientes estaban familiarizados, de tal forma que era después labor suya pensar y decidir cómo podían corregirse (Ruiz Herrero 2015). Sin embargo, al mismo tiempo, todos los textos poseían uno o varios comentarios relacionados con el contenido, que por lo general adoptaban la forma de peticiones de desarrollo de una 
idea, cuestionamientos con contraejemplos de un aspecto de lo manifestado o sugerencias de que se incidiera en una cuestión que no se había abordado. Algunos ejemplos fueron: "¿No piensas que a veces se exagera esa visión melancólica?"; "Me gusta esta visión diferente del problema. ¿No piensas, de todos modos, que la ciudad de Beirut ha perdido parte de su autenticidad?"; "¿Y ahora la gente no lo hace? ¿No crees que se trata más bien de una diferencia entre campo y ciudad?" o "¿Recuerdas esa época? ¿Cuántos años tenías cuando comenzó la guerra?", dirigido este último a uno de los pocos estudiantes que sí que había vivido el periodo.

Así, en la siguiente sesión, aprovechando la realización de la prueba escrita final, a medida que cada uno terminaba, se le hacía entrega del texto revisado, al que se había incorporado una cuartilla grapada en la parte superior en la que se pedía que respondiera a las anotaciones del profesor. Esta fue la única indicación que se proporcionó, a partir de lo cual resultó de considerable interés comparar y contrastar las diferentes contestaciones que se devolvieron, cada una de las cuales atendía a aquello que se consideraba más pertinente. Así, una estudiante entregó una cuartilla convenientemente numerada en la que respondía de forma puntual a cada una de las observaciones, las de forma y las de fondo, proporcionando una breve explicación del error corregido o de la observación que se incorporaba. Otro estudiante obvió por completo las marcas relativas a errores de forma para embarcarse en una apasionada respuesta acerca de cómo la ciudad de Beirut había superado siglos de violencia y destrucción. Por último, una tercera estudiante pasó revista a su propio proceso de escritura justificando por qué había decidido omitir determinada información y pasándola a desarrollar después (Anexo 2). Por añadidura, y es aquí donde procede incidir, en todos los casos la apertura de un canal de comunicación directo acerca de lo que se había expresado en un primer momento condujo a un intercambio significativo en el que, sin proporcionar pautas de duración o extensión, se elaboraron respuestas elaboradas motivadas por una intención genuina de explicitar las ideas y negociar el significado.

\subsection{Secuencia 2: un foro de discusiones}

La segunda secuencia se planteó en un grupo de ocho personas de un nivel B2. En este caso se incluía en una unidad temática que tenía como objetivo principal la expresión de la opinión, el acuerdo o desacuerdo y la argumentación. Se desarrolló en dos sesiones (durante una hora en la primera y veinte minutos en la segunda) e incorporaba asimismo una propuesta de trabajo para casa.

En la primera sesión se regresa a la pared de la clase como soporte físico de la actividad, si bien en este caso adoptará la forma de un foro de Internet en el que diferentes usuarios presentarán opiniones y reaccionarán con sus comentarios. Se comienza, así, distribuyendo a lo largo de las paredes y las diferentes superficies verticales practicables con las que cuente la clase recortes lo suficientemente grandes donde se puedan leer un amplio repertorio de opiniones potencialmente polémicas. A la hora de seleccionarlas, se trata de elegir cuestiones que se sepa que poseen una cierta capacidad para suscitar disensión en el grupo meta, acaso vinculadas con la actualidad, y que se distribuyan a lo largo de un variado espectro temático, de tal modo que se cubran ámbitos políticos, sociales, culturales y deportivos y que se mezclen reflexiones de carácter más abstracto e ideológico con otras más concretas, prácticas e inmediatas. De este modo, se trata de favorecer la implicación activa de cada una de las personas del grupo en algunos de los 
debates planteados. Así, por ejemplo, para el grupo de ocho personas que realizó la secuencia se seleccionaron doce opiniones distintas, entre las cuales se incluían "A veces las personas más religiosas son las más egoístas e hipócritas"; "El amor eterno no existe, es una fábula romántica"; "Una mujer nunca llegará a ser Presidenta de Líbano"; "La música libanesa actual está en total decadencia"; "La única lengua de Líbano es el árabe y hay que protegerla y promoverla" o "No se debe mantener relaciones sexuales antes del matrimonio". Puesto que la diversidad y la proximidad constituyen los criterios temáticos fundamentales, resulta prioritario manipular o renovar totalmente el repertorio en función de cada grupo meta.

Cada estudiante recibe tres folios divididos horizontalmente en cuatro espacios, el primero de los cuales ocupa la mitad de la hoja, mientras que la parte inferior aparece repartida en los otros tres. Se les informa de que durante la siguiente hora tendrán que responder a tres de las opiniones que se encuentran en las paredes escribiendo en el espacio más grande su reacción, mostrando de forma argumentada su acuerdo o desacuerdo. Una vez que lo hayan hecho colgarán la hoja debajo de la opinión y abrirán así un hilo nuevo en el foro. Además de ello, tendrán que comentar en los espacios inferiores, tres de los hilos iniciados por los compañeros y, en la medida de lo posible, responder a lo que éstos señalen en los hilos que ellos mismos han abierto. La indicación numérica no pretende plantear una pauta rígida que deba cumplirse en el espacio de una hora estimado para este punto de la secuencia. Más bien se trata de plantear una dinámica que ponga en marcha una inercia interactiva que garantice que durante ese periodo se estará escribiendo, independientemente de si se alcanza o no (o se supera) la marca establecida al inicio. Para fomentar la controversia, el profesor puede intervenir en hilos iniciados con comentarios que planteen dudas $o$ disconformidades acerca de lo afirmado por la persona que inició la respuesta y que obliguen a esta a desarrollar su punto de vista en la dirección apuntada. Esta intervención constituye la única que realiza el docente en este punto de la secuencia y que, por lo tanto, se mantiene al nivel del contenido. Se trata de favorecer durante esta hora un espacio de expresión escrita lo más libre y cómodo posible: habrá quien prefiera volver a su asiento para escribir cada texto, quien vaya desplazándose y escribiendo allí donde se encuentra la frase inicial y quien responda directamente sobre el papel colgado de la pared, esto es, una ocupación autónoma y fluida del espacio. Asimismo, si nadie considera que se dificulta su proceso de generación de ideas y textualización, se recomienda reproducir música a un volumen razonable para acompañar todo este paso de la secuencia.

Una vez el tiempo agotado, se recogen todas las muestras, que se analizarán para preparar las dos actividades de la siguiente sesión. A partir de la respuesta a los textos se realizarán dos planteamientos diferentes. El primero, una actividad centrada en la forma de carácter presencial que podría denominarse "El museo de los errores". Se revisarán, pues, los textos y se seleccionará un número de frases determinado que presente cuestiones de carácter léxico, sintáctico, morfológico o de puntuación que resulten de interés particular. Resulta particularmente pertinente seleccionar aquellas cuya corrección requiera la movilización de funciones o estructuras trabajadas en la presente unidad o en momentos anteriores del curso. A cada una se le asigna un número- una cifra entre doce y quince parece razonable-, se imprime de forma separada y se vuelve a colocar en la pared, que ahora se convierte en una exposición de frases. Se propone trabajar por parejas y se reparte a cada una de ellas una plantilla con un espacio 
previsto y numerado para cada frase. Cada pareja dispondrá de unos diez minutos para dar la vuelta a la clase, decidir cuál es la cuestión problemática en cada caso y escribir la alternativa correcta, después de lo cual se procede a la puesta en común. Este tipo de dinámicas presenta el riesgo de que la corrección acabe resultando indiscriminada y centrándose en los aspectos menos sospechados. Es por ello recomendable limitar el número de errores a uno por frase (manipulando, si es necesario, la muestra original), dejar clara esta limitación a la hora de dar las instrucciones y plantear eventualmente un código cromático que señale en cada caso qué tipo de error hay que buscar.

La segunda propuesta está centrada en el contenido y se plantea como una propuesta de expresión escrita para casa que desarrolle el trabajo realizado en la primera sesión. Así, se seleccionará para cada persona dos de los hilos producidos en el primer punto de la secuencia. Uno de ellos será uno de los iniciados por esa misma persona, a la que se pedirá que responda a todas las contribuciones realizadas en la parte inferior del folio, en una hoja aparte. Otro será un hilo en el que no hubiera contribuido durante la primera sesión y en el que se le invitará a responder tanto a la opinión que constituía el punto de partida, la reacción que lanzaba el hilo como los comentarios posteriores. Se subrayará que el formato será el mismo de respuesta de foro, con lo que se indicará que deberán dirigirse a cada uno de los interlocutores directamente. Nuevamente se tratará de seleccionar hilos en los que hayan surgidos puntos de oposición y debate suficientes como para sustentar nuevos intercambios, que pueden reforzarse con comentarios por parte del docente contradiciendo las ideas expuestas o planteando cuestiones relacionadas. Estas, en todo caso, serían las únicas intervenciones registradas en los hilos originales, que no presentarían ningún tipo de corrección de forma explícita o implícita. Quedaría, por último, a criterio del docente que tipo de respuesta- centrada en la forma, en el contenido o mixta- se daría a los textos entregados en la siguiente sesión, si bien tampoco parece razonable extender as infinitum el desarrollo de la producción más allá de un punto en el que la secuencia dé muestras de agotamiento.

\section{Conclusiones}

A partir de lo anterior, cabría subrayar hasta qué punto se puede favorecer el desarrollo de la fluidez en la expresión e interacción escritas incidiendo en la retroalimentación centrada en el contenido, que constituye una valiosa pauta para enfocar la respuesta que el docente proporciona a los escritos. Sin necesidad de plantear la exclusión de la información centrada en la forma, se aboga por la inclusión frecuente y la focalización recurrente en las anotaciones que se concentran en lo que se ha escrito, en las ideas verbalizadas y compartidas. Se trata en parte de contribuir a una cierta desacralización del planteamiento de la destreza en el aula de lengua, reforzando la idea de que fundamentalmente ha de centrarse en la construcción de significado y el intercambio de ideas y experiencias, por encima de cualquier preocupación formalista. Asimismo, se apunta la conveniencia de alterar el papel tradicional del docente en el proceso de escritura, que deja de sancionar la corrección de unas formas plasmadas en papel para convertirse en interlocutor que, además de propiciar posibles mejoras organizativas o lingüísticas del texto en cuestión, se compromete mostrando interés en un diálogo. Pero, ante todo, si conviene ensalzar el papel de este tipo de respuesta para favorecer la producción de un caudal más rico y sostenido es porque al centrar nuestra atención en lo que se transmite más que en el cómo en última instancia dignificamos la opinión, la 
experiencia o la imaginación de quien escribe, a quien se le da a entender que a la hora de escribir en el aula no solo está cumpliendo con un trámite académico, sino que está participando en un intercambio comunicativo potencialmente significativo. No se trata, pues, - o no ha de tratarse- de una mera excusa para manipular estructuras gramaticales sino de una oportunidad para organizar vivencias, crear historias o compartir ideas con otros individuos.

\section{Bibliografía}

ABDEL LATIF, Muhammad (2014). Recent developments in writing fluency measurement. En Exploring EFL Fluency in Asia (pp.196-209). Londres: Palgrave Macmillan.

DORMER, Robert (2016). «Fluency' in L2 Writing: A Literature Review». Kwansei Gakuin University Humanities Review, 21, 275-284.

Ellis, Rod, Gary BARKHUIZEN (2005). Analysing learner language. Oxford: Oxford University Press.

FERRIS, Dana (1999). "The case for grammar correction in L2 writing classes: A response to Truscott». Journal of second language writing, 8,1, 1-11.

FrASER, Sue (2014). «Assessing Fluency: A Framework for Spoken and Written Output». En Exploring EFL Fluency in Asia (pp. 178-195). Londres: Palgrave Macmillan.

JENSEN, Eric (1998). Teaching with the brain in mind. ASCP.

LENNON, Paul (2000). «The lexical element in spoken second language fluency». En Perspectives on fluency (pp. 25-42). Michigan: University of Michigan.

Muller, Theron (2014). «Implementing and Evaluating Free Writing in a Japanese EFL Classroom». En Exploring EFL Fluency in Asia (pp. 163-177). Londres: Palgrave Macmillan.

NAtion, Paul (2014). «Developing fluency». En Exploring EFL Fluency in Asia (pp. 25-42). Londres: Palgrave Macmillan.

RAtEY, John J. (2001). A user's guide to the brain: Perception, attention, and the four theatres of the brain. Ney York: Vintage.

RUIZ HERRERO, Juan (2015). «La corrección y revisión de textos mediante códigos de marcas: análisis crítico de una herramienta docente». MarcoELE: Revista de Didáctica Español Lengua Extranjera, 20, p. 12.

SKEHAN, Peter (2009). «Modelling second language performance: Integrating complexity, accuracy, fluency, and lexis». Applied linguistics, 30,4, 510-532.

TRUSCOTT, John (1996). "The case against grammar correction in L2 writing classes». Language learning, 46, 2, 327-369. 


\section{ANEXO 1}

Para practicar tu español, y también porque te gusta ver fotos de gente que no conoces, estás en un grupo de Facebook de hispanoparlantes en Líbano. Hoy una chica ha publicado este mensaje. Léelo y, luego, responde en la parte de abajo. Para darte algunas ideas vamos a ver un vídeo.

\section{Manolita Coliflores}

$2 \mathrm{~h}$.

Llevo un mes en Líbano y hasta ahora todo estupendo: me lo estoy pasando muy bien y la experiencia está siendo muy interesante. Hay una cosa, en todo caso, que me parece muy curiosa. Cuando hablo con gente de aquí, sobre todo si son un poco mayores, siempre dicen que Líbano antes era muy diferente, que las cosas han cambiado mucho y que ahora no tiene nada que ver. ¿Alguien más ha escuchado eso? ¿Sabéis si es verdad o si es un mito un poco absurdo que la gente repite? ¿Hay alguien por aquí que pueda decirnos cómo era Líbano por los años sesenta o setenta del siglo pasado? ¡Muchas gracias!

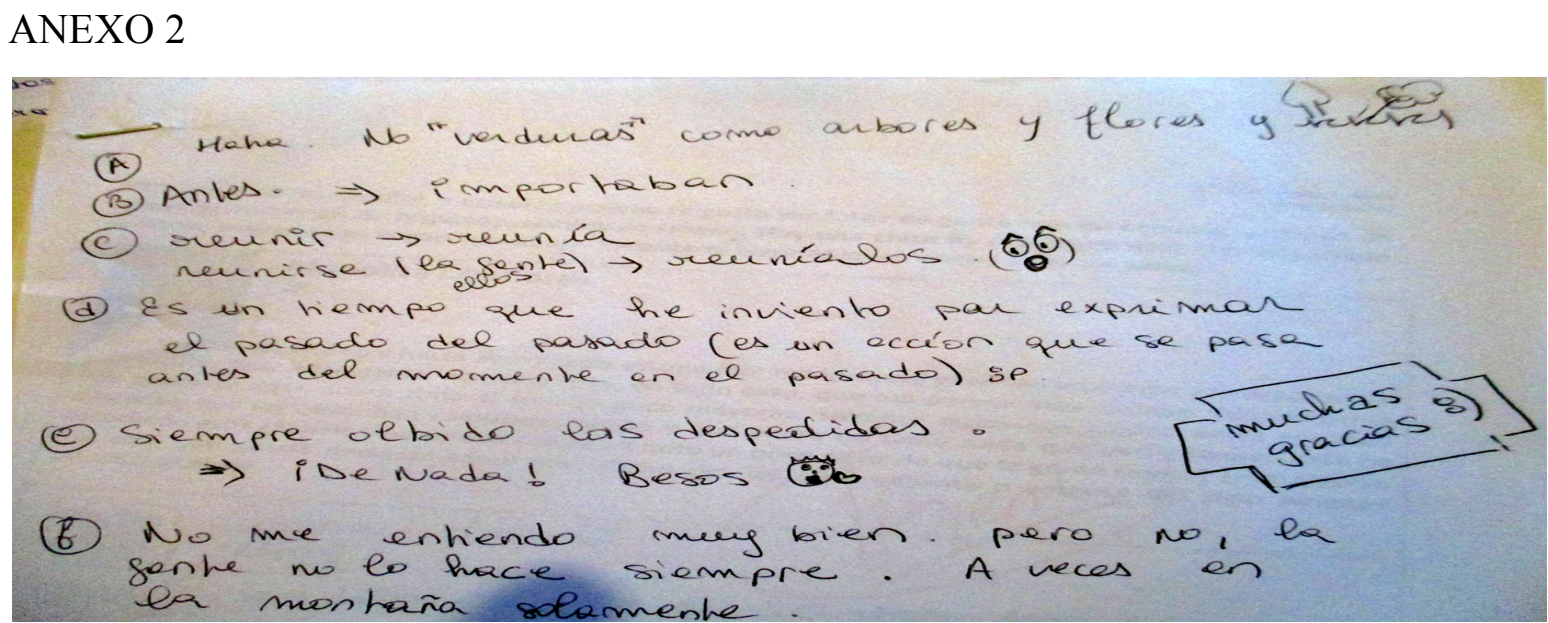




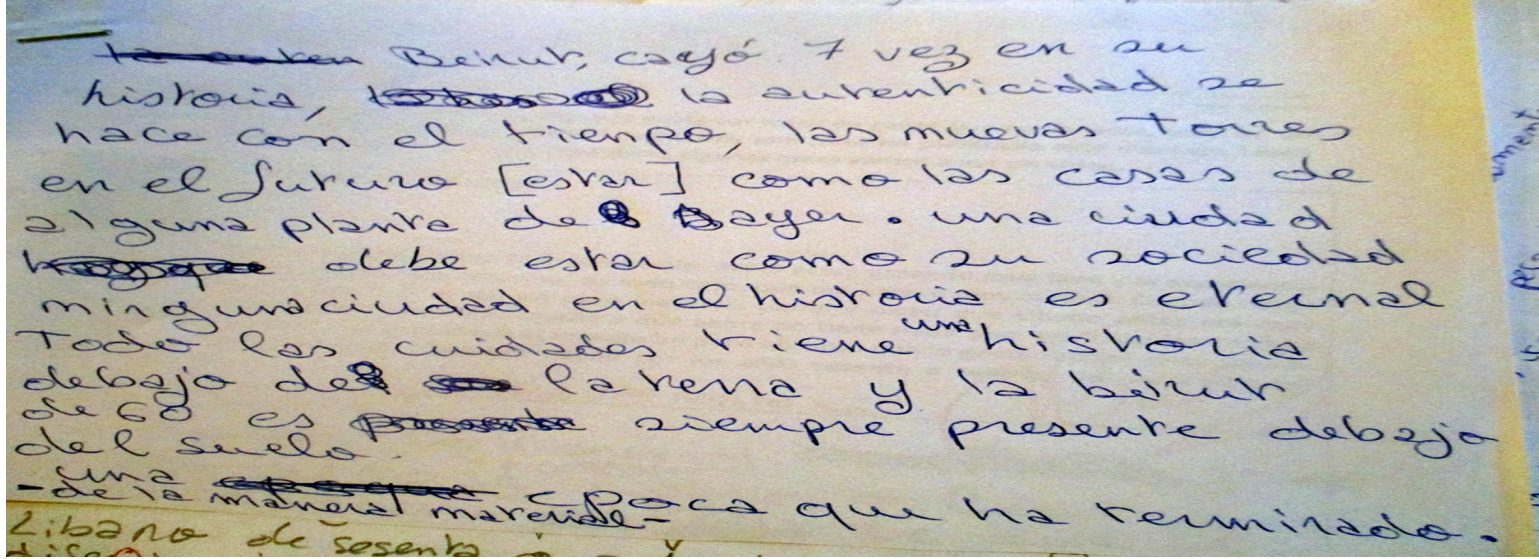

Guité la parte de lostecinas por fue estuve cousada y mo pude pemsar oescribir (Losiemto).

pero chore vol a exribir $f$ completer mi idea: $(:) 1)$ )

Antes eosvecimos se conocíam y visitabam los demás per acea ahora coŕnadie conoce a su vecino y cada unde tieme sa uida. Antes sitemáa un problema tu vecimo podia ayudarte y darte consejos pera soeverlo pero ahora a tu vecimo le gusta verte temer un problema porque no es amable ocariñoso tes muy ezásta. 